\title{
ASYMPTOTIC DESCRIPTION OF MAXIMUM MISTUNING AMPLIFICATION OF BLADED DISK FORCED RESPONSE
}

\author{
Carlos Martel \\ E.T.S.I. Aeronáuticos \\ Universidad Politécnica de Madrid \\ 28040 Madrid, Spain \\ carlos.martel@upm.es
}

\author{
Roque Corral* \\ Industria de Turbopropulsores S.A. \\ 28830 Madrid, Spain \\ roque.corral@itp.es
}

\begin{abstract}
The problem of determining the maximum forced response vibration amplification that can be produced just by the addition of a small mistuning to a perfectly cyclical bladed disk still remains not completely clear. In this paper we apply a recently introduced perturbation methodology, the Asymptotic Mistuning Model (AMM), to determine which are the key ingredients of this amplification process, and to evaluate the maximum mistuning amplification factor that a given modal family with a particular distribution of tuned frequencies can exhibit. A more accurate upper bound for the maximum forced response amplification of a mistuned bladed disk is obtained from this description, and the results of the AMM are validated numerically using a simple mass-spring model.
\end{abstract}

\section{INTRODUCTION}

The term mistuning refers to the small differences among the (nominally designed to be identical) rotor blades in turbomachinery bladed disks. These small unavoidable imperfections typically result from the manufacturing process and wear, and break the cyclic symmetry of the structure, splitting the tuned eigenvalue pairs and preventing the existence of pure travellingwaves as natural vibration modes of the mistuned structure. This loss of cyclic symmetry has a dramatic effect in the forced response of the bladed disk: the resulting mistuned blade vibration patterns are no longer uniformly distributed circumferen-

\footnotetext{
*Also Associate Professor at E.T.S.I. Aeronáuticos, Universidad Politécnica de Madrid, 28040 Madrid, Spain.
}

tially and can exhibit localized vibration amplitudes significantly higher than those of the tuned case. The amplification of the response levels may lead to a severe reduction of the fatigue life of the blades, and thus mistuning has a strong negative impact on safety, operability and readiness. It is therefore very important to understand the basic mechanisms behind mistuning amplification in order to be able to produce new technological solutions that can predict and control its effect, and that can result in an enhancement of overall aircraft readiness and a substantial reduction of maintenance costs.

Numerous studies have been conducted to understand mistuning effects on the dynamics of bladed disc assemblies, see, e. g., the survey paper of Slater et al. [1] and the more recent review by Castanier and Pierre [2], and the exhaustive references therein.

Despite of the great research activity developed in this subject since the 1970s, there are basic questions of critical technological importance such as the determination of the maximum vibration amplification factor of forced response due to mistuning that still remain without a clear answer. Since the seminal work of Whitehead [3] in maximum mistuning amplification to the recent presentations by Han et al. [4,5], there not appears to be a solid consensus about the maximum mistuning amplification factor.

The problem of directly computing the maximum amplification factor is a formidable task because: (i) mistuning breaks the cyclic symmetry of the problem and the whole bladed disk (instead of a single sector) has to be considered, and (ii) the random nature of the mistuning requires the evaluation of the response of

Copyright (c) 2008 by ASME 


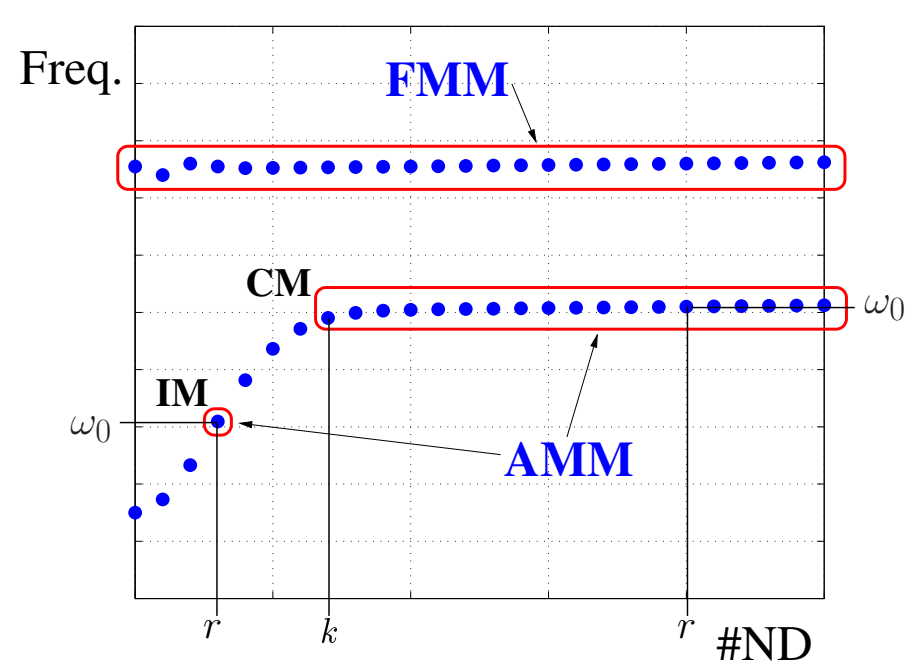

Figure 1. Tuned natural vibration frequencies vs. number of nodal diameters for a bladed-disk. IM: isolated mode, CM: clustered modes.

the system for a large number of different mistuning patterns to somehow statistically explore all possible configurations. Petrov and Ewins [6] formulated the problem of finding the mistuning pattern that gives the maximum amplification factor as an optimization problem. They were able to locate the worst mistuning pattern in a realistic bladed disk configuration that was quite CPU costly, but this direct approach did not provide much insight into the basic mechanisms of mistuning amplification.

In order to overcome these difficulties several types of simplified models have been developed to reduce the required computational time. Simple lumped models with the structure modeled by 1 DOF mass-spring systems (see [7] and the more recent work by [8-10]) and more detailed reduced order models (ROMs) [11-14] yield good results once its coefficients have been appropriately tuned, but do not provide much insight in the basic physical mechanisms involved in the amplification of the forced response due to mistuning.

Recently, a simpler model, known as the Fundamental Mistuning Model (FMM), that reduces the set of nominal modes to a single modal family has been developed [15-17]. This method only requires to know the natural frequencies of the tuned system, but its applicability is limited to a modal family with nearly equal frequencies (see Fig. 1).

This paper presents a further reduction of the FMM for the very frequent case in which all modes of the family do not share the same frequency (see Fig. 1). This new model is derived by means of a fully consistent asymptotic analysis starting from the complete problem of the mistuned bladed disk. The small parameters in the asymptotic expansion are the small frequency corrections induced by mistuning and the small damping of the tuned modes. The resulting model, referred to hereafter as the Asymptotic Mistuning Model (AMM), is not significantly faster than the FMM but allows us to uncover the basic physical mechanisms of mistuning since it accounts only for the minimum subset of degrees of freedom that play a relevant role in the mistuning process. The AMM also provides a more accurate upper bound for the maximum mistuned response amplification that a forced bladed disk can experience.

The AMM has been already used by the authors [18] for the study of the optimal intentional mistuning patterns for the stabilization of aerodynamically unstable rotors. In this paper we describe in detail the derivation of the AMM for the forced response of a general realistic mistuned bladed disk. And we apply this methodology to two frequent modal configurations not covered by the FMM: isolated mode and clustered modes (labeled IM and CM, respectively, in Fig. 1), obtaining the maximum amplification factor and the associated mistuning distribution, and comparing the AMM results with those from the numerical simulation of a mistuned mass-spring model.

\section{NOMENCLATURE}

M Mass matrix.

K Stiffness matrix.

F External forces.

X DOF displacements vector.

TW Travelling wave.

$N, m \quad$ Number of sectors, number of DOF per sector.

$Z_{k j} \quad$ In-sector tuned eigenmode.

P Change of basis matrix from TW to displacements.

A Travelling wave mode amplitude vector.

$\Delta \mathbf{M} \quad$ Mass mistuning matrix.

$\Delta \mathbf{K} \quad$ Stiffness mistuning matrix.

$r, \omega \quad$ Engine order and frequency of the forcing.

$\omega_{0} \quad$ Tuned natural frequency of the excited TW ( $r$ nodal diameters).

$d \quad$ Damping of the tuned natural TW modes.

$N_{a} \quad$ Number of active modes.

$\omega_{a} \quad$ Active mode tuned natural frequency.

$\Delta \quad$ Mistuning matrix in the TW basis.

$D, D_{i j} \quad$ Mistuning coefficients in the TW basis.

$\omega_{p}, \omega_{c} \quad$ Spring frequencies of the model in Fig. 2.

$\varepsilon \quad$ Mistuning size of the model in Fig. 2.

$\delta_{j} \quad$ Mistuning distribution of model in Fig. 2.

$c \quad$ Damping of the model in Fig. 2.

$f \quad$ Forcing amplitude of the model in Fig. 2.

\section{ASYMPTOTIC MISTUNING MODEL}

This methodology is based on the fact that the mistuned bladed disk constitutes just a small distortion of the tuned one, and uses asymptotic perturbation techniques to evaluate the ef- 
fect of the small mistuning in the magnitude of the forced vibration amplitude. The resulting simplified description includes the main physical mechanisms involved in the mistuning amplification process.

\section{Tuned System}

The tuned system can be regarded as the background state on top of which we compute the mistuning correction. In this section we give a rather detailed description of the forced response problem for a general tuned bladed disk. These are all well known results, but we believe it is important to briefly collect them here to clarify the notation before proceeding with the evaluation of the corrections.

The equations of motion for the forced oscillations of a perfectly tuned bladed disk with $N$ identical sectors take the form

$$
\mathbf{M} \ddot{\mathbf{x}}+\mathbf{K x}=\mathbf{f}(t)
$$

where the independent bladed disk degrees of freedom (after an appropriate FEM discretization of the structure) are stored in $\mathbf{x}(t), \mathbf{M}$ and $\mathbf{K}$ are the mass and stiffness matrices (both symmetric and positive definite), and $\mathbf{f}(t)$ accounts for the time dependent external forces acting on the blades. Note that damping has not been included in the above formulation since it is typically a small effect and it will be added later on together with the mistuning corrections.

The natural vibration modes of the system are obtained from Eqn. (1) with $\mathbf{f}(t)$ set to zero, and can be written using complex notation as

$$
\mathbf{x}=\mathbf{X} \mathrm{e}^{\mathrm{i} \omega t}+\text { c.c. }
$$

where the complex vibration mode shape $\mathbf{X}$ and the mode oscillation frequency $\omega$ (c.c. stands for the complex conjugate) are given by the following symmetric generalized eigenvalue problem

$$
\left(\mathbf{K}-\omega^{2} \mathbf{M}\right) \mathbf{X}=\mathbf{0}
$$

If we now divide the response vector as

$$
\mathbf{X}=\left[\begin{array}{c}
X_{1} \\
\vdots \\
X_{j} \\
\vdots \\
X_{N}
\end{array}\right]
$$

with the vector $X_{j}$ containing the displacements of the $m$ degrees of freedom associated with sector $j$, then the eigenvalue problem takes the form

$$
\left(\left[\begin{array}{ccccc}
K & K_{c} & 0 & \cdots & K_{c}^{\mathrm{T}} \\
K_{c}^{\mathrm{T}} & K & K_{c} & \cdots & 0 \\
& & \ddots & \ddots & \\
K_{c} & 0 & \cdots & K_{c}^{\mathrm{T}} & K
\end{array}\right]-\omega^{2}\left[\begin{array}{ccccc}
M & M_{c} & 0 & \cdots & M_{c}^{\mathrm{T}} \\
M_{c}^{\mathrm{T}} & M & M_{c} & \cdots & 0 \\
& & \ddots & \ddots & \\
M_{c} & 0 & \cdots & M_{c}^{\mathrm{T}} & M
\end{array}\right]\right)\left[\begin{array}{c}
X_{1} \\
X_{2} \\
\vdots \\
X_{N}
\end{array}\right]=\left[\begin{array}{c}
0 \\
0 \\
\vdots \\
0
\end{array}\right],
$$

where the $m \times m$ sector stiffness and mass matrices $K$ and $M$ are symmetric and the coupling between adjacent sectors is represented by the coupling stiffness and mass matrices $K_{c}$ and $M_{c}$.

The cyclic symmetry of the bladed disk (that is composed of a sector repeated $N$ times and arranged periodically) is now evident in the matrices in Eqn. (5) that exhibit a block circulant structure, i.e., each row can be obtained from the previous one after a wrap-around forward block shift. The associated complex eigenvectors are of the form

$$
\left[\begin{array}{c}
X_{1} \\
\vdots \\
X_{j} \\
\vdots \\
X_{N}
\end{array}\right]=\left[\begin{array}{c}
Z_{k} \mathrm{e}^{\mathrm{i}\left(\frac{2 \pi k}{N}\right) 1} \\
\vdots \\
Z_{k} \mathrm{e}^{\mathrm{i}\left(\frac{2 \pi k}{N}\right) j} \\
\vdots \\
Z_{k} \mathrm{e}^{\mathrm{i}\left(\frac{2 \pi k}{N}\right) N}
\end{array}\right] \quad \text { for } \quad k=1 \ldots N
$$

and correspond to travelling waves (TW) with wavenumber $k$ (and $\min (|k|,|N-k|)$ nodal diameters) that, taking into account Eqn. (2), rotate with constant angular velocity $\frac{\omega_{k}}{k}$. The complex vector $Z_{k}$ of size $m$ contains the mode shape details and the only eigenvector variation from sector to sector is just a phase shift of amount $\frac{2 \pi k}{N}$ (the so-called "interblade phase angle").

Note that the modes $\mathbf{X}$ of the undamped problem in Eqn. (2) can be taken to be real but instead we take them complex (see Eqn. (6)) in order to use the much simpler sector-to-sector TW formulation.

If we now insert expression (6) into Eqn. (5), $N$ decoupled eigenvalue problems of size $m$ are obtained

$$
\begin{gathered}
\left(\left(K_{c}-\omega^{2} M_{c}\right) \mathrm{e}^{\mathrm{i}\left(\frac{2 \pi k}{N}\right)}+\left(K_{c}-\omega^{2} M_{c}\right)^{\mathrm{T}} \mathrm{e}^{-\mathrm{i}\left(\frac{2 \pi k}{N}\right)}+K-\omega^{2} M\right) Z_{k}=0 \\
\text { for } k=1 \ldots N
\end{gathered}
$$

which, for each $k$, have $m$ real eigenvalues, $\omega_{k 1}^{2}, \ldots, \omega_{k m}^{2} \geq 0$, that give the oscillation frequencies of the structure. The associated eigenvectors, $Z_{k 1}, \ldots, Z_{k m}$, are, in general, complex and can be normalized in such a way that the square matrix

$$
P_{k}=\left[Z_{k 1}\left|Z_{k 2}\right| \ldots \mid Z_{k m}\right]
$$


verifies

$$
\begin{gathered}
P_{k}^{\mathrm{H}}\left(M_{c} \mathrm{e}^{\mathrm{i}\left(\frac{2 \pi k}{N}\right)}+M_{c}^{\mathrm{T}} \mathrm{e}^{-\mathrm{i}\left(\frac{2 \pi k}{N}\right)}+M\right) P_{k}=I \text { and } \\
P_{k}^{\mathrm{H}}\left(K_{c} \mathrm{e}^{\mathrm{i}\left(\frac{2 \pi k}{N}\right)}+K_{c}^{\mathrm{T}} \mathrm{e}^{-\mathrm{i}\left(\frac{2 \pi k}{N}\right)}+K\right) P_{k}=\Omega_{k}^{2}=\left[\begin{array}{ccc}
\omega_{k 1}^{2} & & 0 \\
& \ddots & \\
& & \omega_{k m}^{2}
\end{array}\right](10) \\
\text { for } k=1 \ldots N,
\end{gathered}
$$

that is, the eigenvectors are orthonormal with respect to the mass matrix and turn into diagonal form the sector stiffness matrix (the superindex ${ }^{\mathrm{H}}$ stands for the conjugate transpose matrix), see e.g. [19].

The eigenvalue equation Eqn. (7) remains invariant under the changes

$$
k \rightarrow N-k, \quad \omega_{k}^{2} \rightarrow \omega_{N-k}^{2} \quad \text { and } \quad Z_{k} \rightarrow \bar{Z}_{N-k},
$$

and thus, for every travelling wave rotating in one direction, there is also another identical one that rotates in the opposite direction with the same velocity.

In summary, the natural vibration modes of the tuned system are travelling wave modes that come in counter-propagating pairs, except for those associated with $k=0$ and $k=\frac{N}{2}$ (present only for $N$ even), which have real $Z_{k}$ and can be seen as nonpropagative standing waves. Property (11) implies that the plot of the natural frequencies versus the number of nodal diameters, $k$, is symmetric (i.e., $\omega_{k}=\omega_{N-k}$ ), and, typically, only its first half (interblade phase angle from 0 to $\pi$ ) is normally represented, as in Fig. 1.

We now go back to the forced problem (1) and consider a travelling wave excitation with frequency $\omega$ and engine order $r$

$$
\mathbf{f}(t)=\mathbf{F e}^{\mathrm{i} \omega t}+\text { c.c. }=\left[\begin{array}{c}
F \mathrm{e}^{\mathrm{i}\left(\omega t+\left(\frac{2 \pi r}{N}\right) 1\right)} \\
\vdots \\
F \mathrm{e}^{\mathrm{i}\left(\omega t+\left(\frac{2 \pi r}{N}\right) j\right)} \\
\vdots \\
F \mathrm{e}^{\mathrm{i}\left(\omega t+\left(\frac{2 \pi r}{N}\right) N\right)}
\end{array}\right]+\text { c.c. },
$$

which is of particular relevance in this context. The response of the system is much more clearly analyzed if we use the basis of the natural travelling wave modes of the system

$$
\mathbf{X}=\mathbf{P A},
$$

with

$$
\mathbf{P}=\frac{1}{\sqrt{N}}\left[\begin{array}{ccc}
P_{1} \mathrm{e}^{\mathrm{i}\left(\frac{2 \pi 1}{N}\right) 1} & \ldots & P_{N} \mathrm{e}^{\mathrm{i}\left(\frac{2 \pi N}{N}\right) 1} \\
\vdots & & \vdots \\
P_{1} \mathrm{e}^{\mathrm{i}\left(\frac{2 \pi 1}{N}\right) j} & \ldots & P_{N} \mathrm{e}^{\mathrm{i}\left(\frac{2 \pi N}{N}\right) j} \\
\vdots & & \vdots \\
P_{1} \mathrm{e}^{\mathrm{i}\left(\frac{2 \pi 1}{N}\right) N} & \ldots & P_{N} \mathrm{e}^{\mathrm{i}\left(\frac{2 \pi N}{N}\right) N}
\end{array}\right] \quad \text { and } \quad \mathbf{A}=\sqrt{N}\left[\begin{array}{c}
A_{1} \\
\vdots \\
A_{j} \\
\vdots \\
A_{N}
\end{array}\right] \text {, }
$$

where the travelling wave modes are the columns of $\mathbf{P}$ (see Eqs. (6) and (8)) and A contains their amplitudes. In the TW basis, the forced problem takes now the diagonal form

$$
\left[\begin{array}{ccc}
\Omega_{1}^{2}-\omega^{2} I & \ldots & 0 \\
\vdots & \ddots & \vdots \\
0 & \ldots & \Omega_{N}^{2}-\omega^{2} I
\end{array}\right]\left[\begin{array}{c}
A_{1} \\
\vdots \\
A_{N}
\end{array}\right]=\left[\begin{array}{c}
0 \\
\vdots \\
P_{r}^{H} F \\
\vdots \\
0
\end{array}\right]
$$

and its solution is very simple:

$$
\begin{array}{r}
A_{i}=0 \text { for } i \neq r \quad \text { and } \\
A_{r}=\left[\begin{array}{c}
A_{r 1} \\
\vdots \\
A_{r m}
\end{array}\right]=\left[\begin{array}{c}
Z_{r 1}^{H} F /\left(\omega_{r 1}^{2}-\omega^{2}\right) \\
\vdots \\
Z_{r m}^{H} F /\left(\omega_{r m}^{2}-\omega^{2}\right)
\end{array}\right]
\end{array}
$$

that is, the response of the tuned undamped system is a travelling wave similar to the forcing, with frequency $\omega$ and $r$ nodal diameters, that peaks when the forcing frequency $\omega$ is equal to any of the natural frequencies $\omega_{r 1}, \ldots, \omega_{r m}$ corresponding to $r$ nodal diameters. Finally, if we also take into account a small damping $d$ (small as compared with the vibration frequency) in the description, then the peak amplitude of each of the travelling waves becomes finite

$A_{r k}=Z_{r k}^{H} F /\left(\omega_{r k}^{2}-\omega^{2}+2 \mathrm{i} d \omega_{r k}\right), \quad$ and $\quad\left|A_{r k}\right| \max =\left|Z_{r k}^{H} F\right| /\left(2 d \omega_{r k}\right)$,

where the damping $d$ has been appropriately included in the above expression in order to have a temporal behavior for the unforced damped natural mode of the form $\mathrm{e}^{\left(-d+\mathrm{i} \omega_{r k}\right) t}$ (note that we make use of the fact that the damping is small as compared with the frequency to neglect second order corrections). The forced response problem is a linear problem, and therefore we can conveniently normalize the magnitude of the maximum tuned response of a given mode to be 1 simply by setting the amplitude of the forcing to be $Z_{r k}^{H} F=2 \mathrm{i} d \omega_{r k}$. 


\section{Mistuning effects.}

The forced response for a mistuned bladed disk is given by

$$
\left((\mathbf{K}+\Delta \mathbf{K})-\omega^{2}(\mathbf{M}+\Delta \mathbf{M})\right) \mathbf{X}=\mathbf{F},
$$

which are simply the tuned equations (3) with the forcing (12) and the mistuning stiffness, $\Delta \mathbf{K}$, and mass, $\Delta \mathbf{M}$, matrices added. $\Delta \mathbf{K}$ and $\Delta \mathbf{M}$ are block diagonal matrices that contain the deviations from the mean stiffness $K$ and mass $M$ sector matrices (see Eqn. (5)), that is,

$$
\begin{gathered}
\Delta \mathbf{K}=\left[\begin{array}{cccc}
\Delta K_{1} & 0 & \cdots & 0 \\
0 & \Delta K_{2} & \cdots & 0 \\
& & \ddots & \\
0 & 0 & 0 & \Delta K_{N}
\end{array}\right], \Delta \mathbf{M}=\left[\begin{array}{cccc}
\Delta M_{1} & 0 & \cdots & 0 \\
0 & \Delta M_{2} & \cdots & 0 \\
& & \ddots & \\
0 & 0 & 0 & \Delta M_{N}
\end{array}\right], \\
\text { with } \quad \sum_{j=1}^{N} \Delta K_{j}=0, \sum_{j=1}^{N} \Delta M_{j}=0, \\
\text { and } \Delta K_{j}=\Delta K_{j}^{\mathrm{T}}, \Delta M_{j}=\Delta M_{j}^{\mathrm{T}} \text { for } \quad j=1 \ldots N .
\end{gathered}
$$

Mistuning of the sector coupling block matrices has not been included in the model above: the coupling matrices involve only a very small subset of the DOF of the sector and, thus, its mistuning is negligible as compared with that of the main sector.

As we did in the previous section, we rewrite Eqn. (18) using the tuned travelling wave mode basis, Eqs. (13) and (14), to obtain

$$
\left(\left[\begin{array}{ccc}
\Omega_{1}^{2}-\omega^{2} I & \ldots & 0 \\
\vdots & \ddots & \vdots \\
0 & \ldots & \Omega_{N}^{2}-\omega^{2} I
\end{array}\right]+\Delta\right)\left[\begin{array}{c}
A_{1} \\
\vdots \\
A_{N}
\end{array}\right]=\left[\begin{array}{c}
0 \\
\vdots \\
P_{r}^{H} F \\
\vdots \\
0
\end{array}\right]
$$

The structure of the mistuning correction matrix in the travelling wave basis

$$
\Delta=\mathbf{P}^{\mathrm{H}}\left(\Delta \mathbf{K}-\omega^{2} \Delta \mathbf{M}\right) \mathbf{P}
$$

can be more easily analyzed if we make use of the Fourier modes of the sector distribution of the mistuning mass and stiffness matrices, $\Delta K_{k}^{\mathrm{F}}$ and $\Delta M_{k}^{\mathrm{F}}$,

$$
\begin{aligned}
& \Delta K_{j}=\sum_{k=1}^{N} \Delta K_{k}^{\mathrm{F}} \mathrm{e}^{\mathrm{i}\left(\frac{2 \pi k}{N}\right) j} \text { and } \\
& \Delta M_{j}=\sum_{k=1}^{N} \Delta M_{k}^{\mathrm{F}} \mathrm{e}^{\mathrm{i}\left(\frac{2 \pi k}{N}\right) j} \text { for } \quad j=1 \ldots N,
\end{aligned}
$$

which verify (see Eqs. (20) and (21))

$$
\begin{gathered}
\Delta K_{N}^{\mathrm{F}}=0, \Delta M_{N}^{\mathrm{F}}=0 \quad \text { and } \quad \Delta K_{k}^{\mathrm{F}}=\overline{\Delta K_{-k}^{\mathrm{F}}}, \Delta M_{k}^{\mathrm{F}}=\overline{\Delta M_{-k}^{\mathrm{F}}}, \\
\Delta K_{k}^{\mathrm{F}}=\left(\Delta K_{k}^{\mathrm{F}}\right)^{\mathrm{T}}, \Delta M_{k}^{\mathrm{F}}=\left(\Delta M_{k}^{\mathrm{F}}\right)^{\mathrm{T}}, \quad \text { for } \quad k=1 \ldots N .
\end{gathered}
$$

After inserting the above expressions into Eqn. (23), the mistuning matrix takes the form

$$
\Delta=\left[\begin{array}{cccc}
0 & \Delta_{12} & \ldots & \Delta_{1 N} \\
\Delta_{21} & 0 & \ldots & \Delta_{2 N} \\
\vdots & \vdots & \ddots & \vdots \\
\Delta_{N 1} & \Delta_{N 2} & \ldots & 0
\end{array}\right]
$$

where each block is related to the Fourier components of the mistuning by

$$
\Delta_{k j}=P_{k}^{\mathrm{H}}\left(\Delta K_{k-j}^{\mathrm{F}}-\omega^{2} \Delta M_{k-j}^{\mathrm{F}}\right) P_{j} .
$$

Note that the mistuning matrix exhibits off diagonal blocks and, thus, mistuning couples tuned travelling waves with different number of nodal diameters. Moreover, according to Eqn. (28), the coupling between the travelling waves with $k$ and $j$ nodal diameters takes place precisely through the $(k-j)$ harmonic of the mistuning.

The effect of small mistuning (and damping) in the response of the system can be computed, in first approximation, as follows. When the forcing frequency (with engine order $r$ ) is close to a resonant tuned frequency $\omega_{0}$ (corresponding to a TW with $r$ nodal diameters), we can distinguish two essentially different types of travelling wave modes:

1. Modes with frequencies that are not close to $\omega_{0}$, that is, modes whose frequency $\omega_{k j}$ is such that $\left|\omega_{0}-\omega_{k j}\right|$ is large as compared with the small mistuning and damping terms. The corresponding equation for this kind of modes in system (22) is of the form

$$
\left(\omega_{k j}^{2}-\omega^{2}\right) A_{k j}=\sum_{i, h=1}^{m} \text { (small terms) } A_{i h}+(\text { small forcing } 9)
$$

where the coefficient in the left hand side is large as compared with the small mistuning, damping and forcing terms in the right hand side because $\omega$ is close to $\omega_{0}$ and thus away from $\omega_{k j}$. After neglecting the small terms, the equation above yields

$$
A_{k j}=0,
$$


that is, all travelling wave modes with frequencies well apart from $\omega_{0}$ do not contribute in first approximation to the mistuning correction.

2. And modes with frequencies close to $\omega_{0}$, i.e., with frequencies that are at a small distance from $\omega_{0}$, of the order of the small damping and mistuning frequency corrections. We will hereafter refer to these modes as "active" modes because they are, in first approximation, the only ones that take part in the mistuned forced response near the resonant frequency $\omega_{0}$. After removing the not active modes of the previous item from Eqn. (22), the resulting equation for an active mode with amplitude $A_{a}$ and tuned structural frequency $\omega_{a}$ reads

$$
\left(\omega_{a}^{2}-\omega^{2}+2 \mathrm{i} d \omega_{0}\right) A_{a}+\sum_{a^{\prime} \neq a} \delta_{a a^{\prime}} A_{a^{\prime}}=F_{a},
$$

where the sum goes over the rest of active modes and $F_{a}=$ $2 \mathrm{i} d \omega_{0}$ for the active mode with $r$ nodal diameters (the one that is being directly forced) and $F_{a}=0$ for the rest. Note that the frequency in the damping and forcing expression can be set, in first approximation, to $\omega_{0}$ for all active modes because the neglected frequency variations just produce higher order corrections. All coefficients in the above equation are small, and, according to Eqn. (28), the mistuning coupling coefficients can be written as

$$
\delta_{a a^{\prime}}=Z_{a}^{\mathrm{H}}\left(\Delta K_{a-a^{\prime}}^{\mathrm{F}}-\omega_{0}^{2} \Delta M_{a-a^{\prime}}^{\mathrm{F}}\right) Z_{a^{\prime}},
$$

where $Z_{a}, Z_{a^{\prime}}, a$ and $a^{\prime}$ are, respectively, the mode shapes and the number of nodal diameters of the active modes $A_{a}$ and $A_{a^{\prime}}$, and the frequency $\omega$ has been approximated by $\omega_{0}$. The mistuning coupling terms verify $\delta_{a a^{\prime}}=\overline{\delta_{a^{\prime} a}}$ because mistuning constitutes a purely structural, conservative perturbation.

In a general situation, we have as many equations like Eqn. (31) as active modes, which form a simplified model for computing the mistuning effect on the forced response. We call this reduced problem the Asymptotic Mistuning Model (AMM) because the above described procedure can be regarded as an asymptotic perturbation method that gives the main (first order) effects of mistuning. The small parameters in this asymptotic expansion are the damping, the mistuning, and the small variation among the natural frequencies of the active modes.

The AMM description retains only the minimal set of travelling wave modes that are involved in the mistuning correction. The simplicity of the AMM reveals that the only TW modes that are relevant are those with frequency close to the natural frequency of the mode that is being forced (i.e. the active modes),
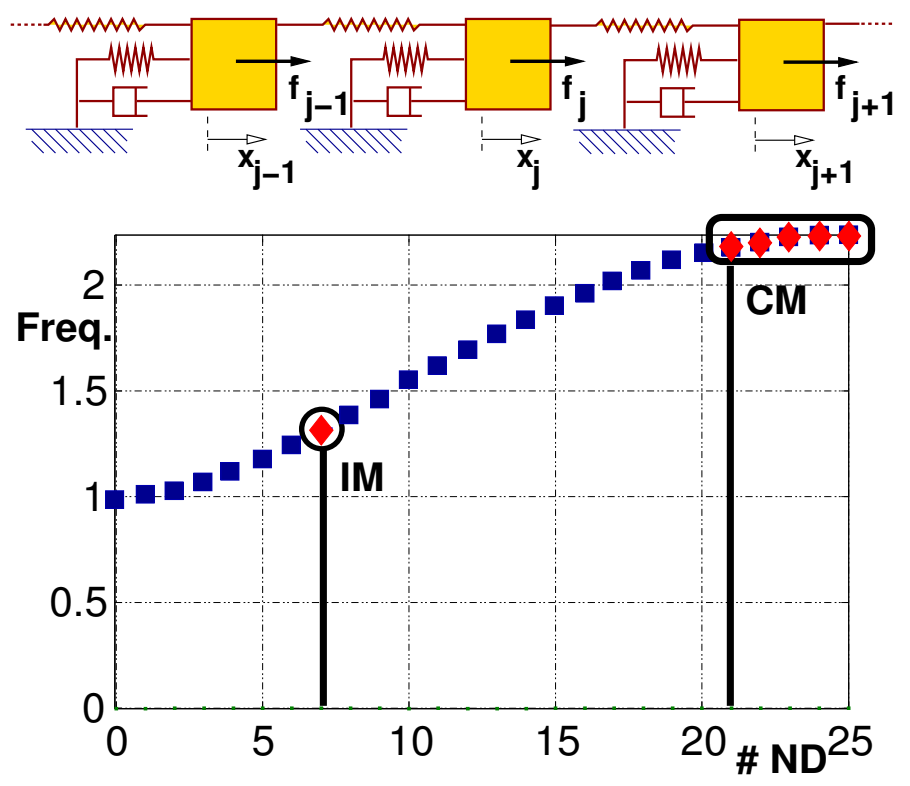

Figure 2. Top: sketch of the simple 1-DOF per sector system. Bottom: tuned frequencies vs. number of nodal diameters (IM: forcing engine order 7, CM: forcing engine order 21).

and that the effect of mistuning is to couple these modes. Moreover, the AMM indicates precisely how this coupling among TWs takes place: it comes from the Fourier mode of the mistuning distribution with wavenumber equal to the difference of the number of nodal diameters of the two TW (see Eqn. (32)).

\section{APPLICATIONS}

In this section we apply the AMM to analyze the mistuning effect on the amplitude of the forced response in the two very frequent modal configurations shown in Fig. 1: isolated modes (IM) and clustered modes (CM).

The results are compared with those obtained from the numerical solution of the simple 1-DOF per sector system sketched in Fig. 2, which is given (in non-dimensional form) by the system of equations

$$
\begin{array}{r}
\ddot{x}_{j}+\omega_{a}^{2}\left(1+\varepsilon \delta_{j}\right) x_{j}+\omega_{c}^{2}\left(2 x_{j}-x_{j+1}-x_{j-1}\right)+c \dot{x}_{j}= \\
=f \mathrm{e}^{\mathrm{i}(\omega t+2 \pi(r / N) j)}+\text { c.c. }, \quad j=1 \ldots N,
\end{array}
$$

where $\omega_{a}^{2}=\omega_{c}^{2}=1, \varepsilon=c=.001, \delta_{j}$ is the mistuning distribution, $f$ is scaled in order to have tuned response equal to 1 , and $N=50$ (see the bottom plot in Fig. 2 for representation of the tuned vibration frequencies of this system). 


\section{Isolated modes}

The TW with $r$ nodal diameters and vibration frequency $\omega_{0}$ labeled IM in Fig. 1 is well apart from the rest, i.e., its distance to the rest of the frequencies is large as compared with the damping and mistuning frequency corrections. In this case, in order to take into account the effect of the mistuning in the response of the system to a forcing with engine order $r$ and frequency close to $\omega_{0}$ it is enough to consider the two counter-rotating TW with frequency $\omega_{0}$ and nodal diameters $\pm r$. In other words, these two modes are the only active modes in the AMM description, which now takes its simplest form

$$
\begin{aligned}
& \left(\omega_{0}^{2}-\omega^{2}+2 \mathrm{i} d \omega_{0}\right) A_{+r}+\bar{\delta} A_{-r}=2 \mathrm{i} d \omega_{0} \\
& \delta A_{+r}+\left(\omega_{0}^{2}-\omega^{2}+2 \mathrm{i} d \omega_{0}\right) A_{-r}=0
\end{aligned}
$$

We can further simplify this system introducing the variable $\Delta \omega=\omega-\omega_{0}$ and neglecting all second order terms to obtain

$$
\left[\begin{array}{cc}
-\Delta \omega+\mathrm{id} & D \\
\bar{D} & -\Delta \omega+\mathrm{id}
\end{array}\right]\left[\begin{array}{c}
A_{+r} \\
A_{-r}
\end{array}\right]=\left[\begin{array}{c}
\mathrm{i} d \\
0
\end{array}\right]
$$

where the effect of mistuning is contained in the off-diagonal coefficient (see Eqn. (32))

$$
D=\delta /\left(2 \omega_{0}\right)=Z_{+r}^{\mathrm{H}}\left(\Delta K_{2 r}^{\mathrm{F}}-\omega_{0}^{2} \Delta M_{2 r}^{\mathrm{F}}\right) \overline{Z_{+r}} /\left(2 \omega_{0}\right),
$$

which involves only the $2 r$ Fourier coefficient of the mistuning distribution.

The response of the system is, in first approximation, just a combination of the two active modes,

$X_{j}=\left(Z_{+r} A_{+r} \mathrm{e}^{\mathrm{i}(2 \pi r / N) j}+Z_{-r} A_{-r} \mathrm{e}^{-\mathrm{i}(2 \pi r / N) j}\right), \quad$ for $\quad j=1, \ldots, N$,

and the amplitude of the displacements is proportional to

$$
\text { Amplitude } \propto\left|A_{+r}+A_{-r}\right| .
$$

This amplitude is equal to 1 for the tuned case and, when mistuning is present, it is given by

$$
\text { Amplitude }=\frac{|(-\Delta \omega+\mathrm{i} d)-D|}{\left.\left|(-\Delta \omega+\mathrm{i} d)^{2}-\right| D\right|^{2} \mid}
$$

The maximum amplitude of the forced response for each mistuned size $|D|$ is plotted in Fig. 3. As expected, the maximum amplitude first grows and then decays when the mistuning size is

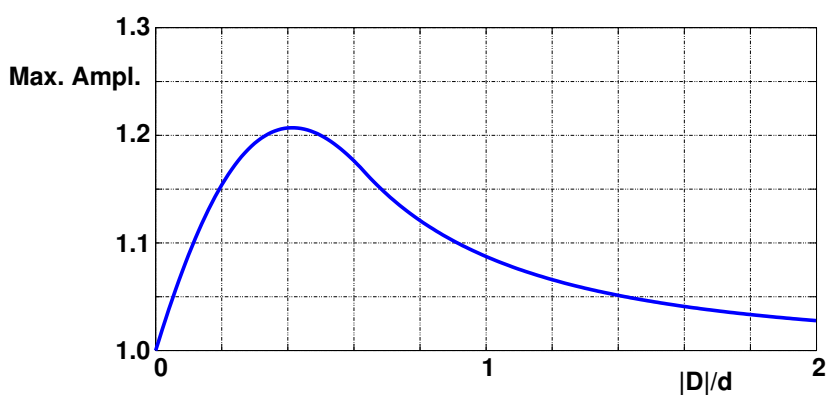

Figure 3. Isolated mode case: maximum attainable amplitude vs. mistuning amplitude $|D|$.

increased. The global maximum attainable mistuned amplitude can be explicitly computed from the above expression to be

$$
\begin{gathered}
\text { Amplitude }_{\max }=\frac{1+\sqrt{2}}{2}=1.207 \ldots \\
D_{\max }=-d(\sqrt{2}-1) \mathrm{i} \\
\Delta \omega_{\max }=0 .
\end{gathered}
$$

These same results were already obtained by Macbain and Whaley [20], which used a formulation for the forced response that included just two forced oscillators with close frequencies and small damping.

In order to check the above results several simulations of the full mass-spring system given by Eqn. (33) have been performed with engine order $r=7$ and with frequency close to the isolated mode frequency labeled IM in Fig. 2. This situation corresponds to an isolated mode since the frequency corresponding to $r=7$ is at a distance from the rest of the frequencies that is large as compared with the damping (more precisely, the distance to its closest neighbours measured in terms of the damping is given by $\left|\omega_{r=7}-\omega_{r=8}\right| / d \sim 150$ and $\left.\left|\omega_{r=7}-\omega_{r=6}\right| / d \sim 143\right)$.

In Fig. 4 the mistuning distribution is harmonic with wavenumber $k=2 r=14$ (see Fig. 4 middle and bottom left plots) and the precise size predicted by the AMM for the maximum amplification. The resulting maximum amplitude, $1.2066 \ldots$ (see Fig. 4 top left plot), is approximately that predicted by the AMM, and, as it can be appreciated from the right plot in Fig. 4, the only TW modes involved in the mistuned response are those with wavenumber 7 and -7 . As predicted by the AMM, the response of the system remains almost unchanged if we add more harmonics with random amplitude to the mistuning distribution (see Fig. 5). And finally, if we remove from the mistuning the harmonic component with wavenumber 14 and keep the others, then there is no appreciable amplification of the response and the system behaves as if tuned, generating only the directly forced TW with wavenumber 7 (see Fig. 6) and again confirming the AMM results and assumptions. 

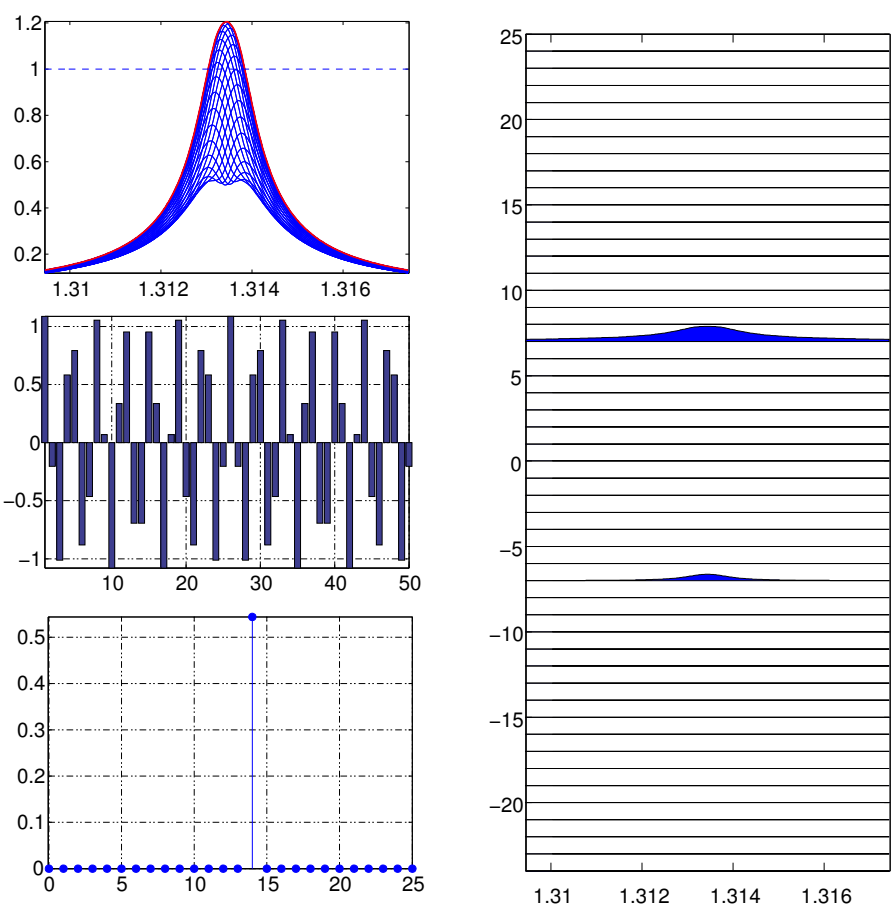

Figure 4. Response of the system in fig. 2 to a forcing with engine order 7 (tuned TW frequency $\omega_{0}=1.3135 \ldots$.) and mistuning pattern composed of a single harmonic with wavenumber 14 (AMM prediction for maximum response). Top left: displacements $\left|x_{j}\right|$ vs. forcing frequency (max $\left.\left|x_{j}\right|=1.2066 \ldots\right)$. Middle left: mistuning distribution $\delta_{j}$. Bottom left: amplitude of the fourier modes of the mistuning distribution. Right: amplitude of the TW components of the response vs. forcing frequency (wavenumber in the vertical axis).

In summary, the mistuning effect in the forcing of a TW with frequency well apart form its neighbors (i.e. with a frequency gap large as compared with the damping) can produce a maximum amplification of up to $20 \%$ approximately, which takes place for a very small mistuning size (of the order of the damping) and through the mistuning harmonic with wavenumber equal to twice the engine order of the forcing. This particular forced response sensitivity to the mistuning harmonics was already noticed by Kenyon and Griffin [21] in their numerical simulations using a simplified ring system, and the maximum factor $\frac{1+\sqrt{2}}{2}$ can be obviously guessed from some of the amplification curves presented in their paper. The systematic approach provided by the AMM goes further than those previous results, giving more precise information and allowing to quantitatively evaluate the maximum attainable amplification factor and the associated mistuning pattern in a general bladed disk configuration.
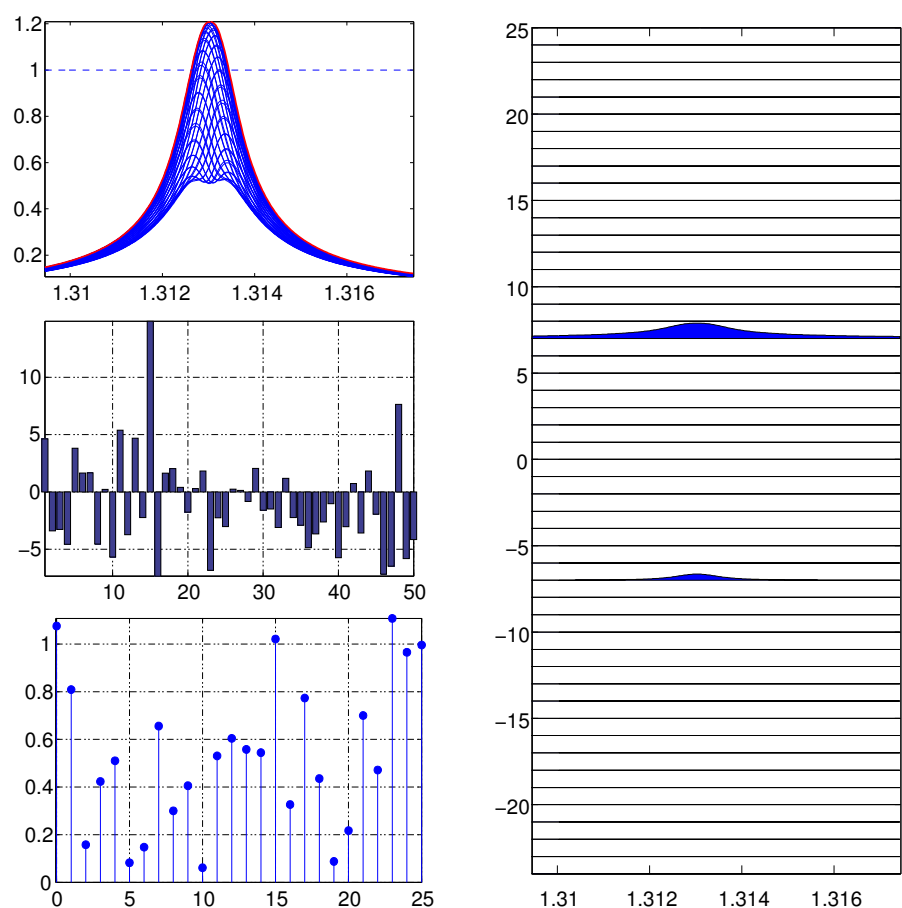

Figure 5. Same as in Fig. 4 but with the rest of the harmonics added to the mistuning pattern with random amplitude $\left(\max \left|x_{j}\right|=1.2085 \ldots\right)$.
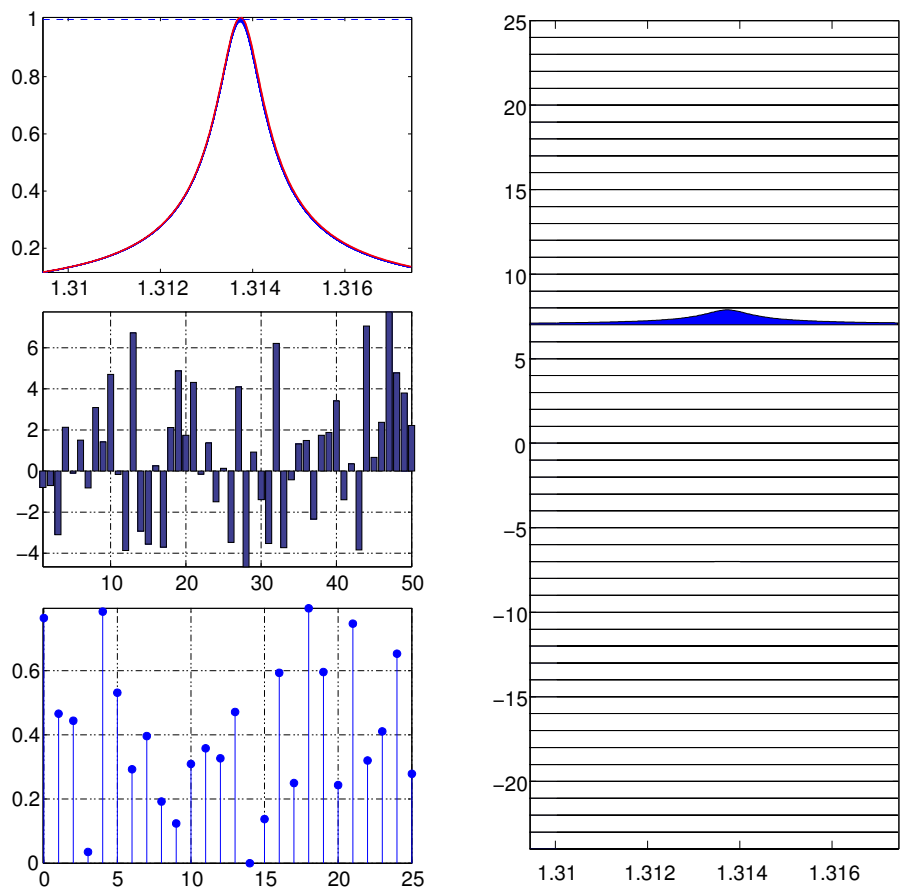

Figure 6. Same as in Fig. 4 but for a mistuning pattern with zero harmonic with wavenumber 14 and the rest with random amplitudes $\left(\max \left|x_{j}\right|=1.0069 \ldots\right)$. 


\section{Clustered modes}

If we now force the system with an engine order $r$ such that the corresponding tuned TW has a frequency $\omega_{0}$ on the horizontal part of the frequency distribution of the modal family (see the encircled modes labeled CM in Fig. 1), then we have more that two active modes. The AMM description, according to Eqn. (31), can now be written as

$$
\left[\begin{array}{ccccc}
d_{k} & & & & \\
& d_{k+1} & & & \\
& & \ddots & & \\
& & & & \\
& D^{\mathrm{H}} & & d_{-(k+1)} & \\
& & & & d_{-k}
\end{array}\right]\left[\begin{array}{c}
A_{k} \\
A_{k+1} \\
\vdots \\
A_{-(k+1)} \\
A_{-k}
\end{array}\right]=\left[\begin{array}{c}
0 \\
\vdots \\
\mathrm{i} d \\
\vdots \\
0
\end{array}\right]
$$

where we have ordered the active modes consecutively in the number of nodal diameters (recall that it has to be considered only modulo $N$ ). The group of active modes are those with $k$ or more nodal diameters (see Fig. 1), and we thus have a total of $N_{a}=N-2 k+1$ active modes, where $N$ is the number of sectors of the bladed disk. The diagonal elements of the above matrix are of the form

$$
d_{j}=\Delta \omega_{j}-\Delta \omega+\mathrm{i} d,
$$

where $\Delta \omega_{j}=\omega_{j}-\omega_{0}, \Delta \omega=\omega-\omega_{0}$, and we have again neglected higher order corrections. Making use of Eqn. (32), the sub and super-diagonal mistuning terms ( $D$ in the matrix above) are readily seen to be

$$
D_{j j^{\prime}}=Z_{j}^{\mathrm{H}}\left(\Delta K_{j-j^{\prime}}^{\mathrm{F}}-\omega_{0}^{2} \Delta M_{j-j^{\prime}}^{\mathrm{F}}\right) Z_{j^{\prime}} /\left(2 \omega_{0}\right)
$$

and the forcing terms are all zero except that associated with the TW with $r$ nodal diameters.

If one takes into account that, in the almost constant frequency region that we are describing, the different mode shapes are approximately equal except for the different interblade phase angle, then $Z_{j}=Z$ (independent of $j$ and real, see Eqn. (11)), and the above expression can be further simplified to

$$
D_{j j^{\prime}}=Z^{\mathrm{H}}\left(\Delta K_{j-j^{\prime}}^{\mathrm{F}}-\omega_{0}^{2} \Delta M_{j-j^{\prime}}^{\mathrm{F}}\right) Z /\left(2 \omega_{0}\right)=D_{j-j^{\prime}},
$$

which depends only on the difference $\left(j-j^{\prime}\right)$ and allows us to rewrite the AMM as

$$
\left[\begin{array}{ccccc}
\frac{d_{k}}{\overline{D_{1}}} & d_{1} & D_{2} & \ldots & D_{N_{a}-1} \\
& & D_{1} & \ldots & D_{N_{a}-2} \\
\overline{D_{N_{a}-2}} & \ldots & & d_{-(k+1)} & D_{1} \\
\overline{D_{N_{a}}-1} & \ldots & & \overline{D_{1}} & d_{-k}
\end{array}\right]\left[\begin{array}{c}
A_{k} \\
A_{k+1} \\
\vdots \\
A_{-(k+1)} \\
A_{-k}
\end{array}\right]=\left[\begin{array}{c}
0 \\
\vdots \\
\mathrm{i} d \\
\vdots \\
0
\end{array}\right]
$$

Note that the only harmonics of the mistuning distribution that play a role in the mistuned forced response are $D_{1}, D_{2}, \ldots, D_{N_{a}-1}$, which couple different active modes.

The AMM is in this case a $N_{a} \times N_{a}$ linear system, and in order to compute the maximum mistuned forced response we have to locate the mistuning coefficients $D_{1}, D_{2}, \ldots, D_{N_{a}-1}$ that maximize the amplitude of the displacement

$$
\text { Amplitude } \propto\left|A_{k}+A_{k+1}+\cdots+A_{-(k+1)}+A_{-k}\right| .
$$

In this case we have not been able to solve explicitly this problem, but an upper bound for the maximum response can be obtained just by using the same argument as in Whitehead $[3,22]$. If we premultiply Eqn. (47) by the row vector $\left[\bar{A}_{k}, \bar{A}_{k+1}, \ldots, \bar{A}_{-(k+1)}, \bar{A}_{-k}\right]$ and substract the complex conjugate, we arrive to the following equation

$$
\left|A_{k}\right|^{2}+\left|A_{k+1}\right|^{2}+\cdots+\left|A_{-(k+1)}\right|^{2}+\left|A_{-k}\right|^{2}=\operatorname{Re}\left(A_{r}\right),
$$

which simply states that the work of the external forcing is equal to the energy dissipated by the damping. Now we can maximize the amplitude in Eqn. (48) subjected only to the work equation (49) constrain. This problem is completely similar to that solved in Whitehead [3,22] but with $N$ chaged by $N_{a}$, and therefore the resulting response amplitude is at most

$$
\text { Amplitude }_{\max }=\frac{1+\sqrt{N_{a}}}{2} .
$$

Now, in order to locate the mistuning distribution that produces the maximum amplitude, we have to numerically solve the optimization problem given by the simplified AMM formulation, which, due to its reduced size, does not require large scale computational resources. Note that the number of variables in the optimization in the worst case when all family modes are active $\left(N_{a}=N\right)$ is $N$ : the number of harmonics of the mistuning distribution (which is real and with average 0) plus the forcing frequency.

The AMM formulation has been used to describe the mistuned forced response of the mass-spring system given by 
Eqn. (33) with a forcing engine order $r=21$ and with a frequency close to the frequency of the clustered modes labeled $\mathrm{CM}$ in Fig. 2. We have to take into account also the symmetric TW with negative wavenumber and thus the cluster in Fig. 2 actually contains 9 TW modes, with wavenumbers: $21,22,23,24,25,-24,-23,-22,-21$.

The AMM indicates that the only relevant harmonics are those that couple the 9 active modes, that is $D_{1}, \ldots, D_{8}$ (recall that $D_{i}=\bar{D}_{-i}$ because the mistuning is a real pattern). We have solved numerically the AMM optimization problem with a number of active modes $N_{a}=9$ and the corresponding tuned frequencies from the clustered modes in Fig. 2. The resulting mistuning pattern (with only the harmonics $D_{1}, \ldots, D_{8}$ ) has been then inserted in the full mass-spring model given by Eqn. (33) and the response is shown in Fig. 7. The maximum amplitude response is $1.991 \ldots$, in close agreement with the AMM predicted value $\frac{1+\sqrt{9}}{2}=2$, and the only TW modes of the response that are nonzero are those with wavenumbers $21,22, \ldots,-22,-21$ (the AMM active modes). In order to confirm that the only harmonics of the mistuning distribution that affect the response are those with wavenumbers $1,2, \ldots, 8$, we add to the mistuning the rest of the harmonics with random amplitude and, as it can be seen in Fig. 8, the response of the system is practically unaltered. Finally, the response shown in Fig. 9 corresponds to removing only the mistuning harmonics with wavenumber $1,2, \ldots, 8$ and, again as predicted by the AMM, the system simply does not feel the effect of the remaining mistuning harmonics. There is almost no amplification with respect to the tuned system, and no coupling among the TW: the only TW mode of the response that is nonzero is that with wavenumber 21 (the one that is being directly excited by the external forcing).

It is also interesting to mention that expression (50) can be also validated with the numerical optimization results presented by Petrov and Ewins [6] for a realistic high pressure turbine bladed disk with $N=92$ sectors. They obtained a maximum amplification factor of 5.02, and that resulting from Whitehead [3,22] expression is $(1+\sqrt{N}) / 2=5$.30. From Fig. 10 of Petrov and Ewins [6] it can be estimated that for a sixth engine order excitation the number of active modes is $N_{a}=83$ (all modes in the family with 5 or more nodal diameters), and using (50) the resulting amplification factor is $\left(1+\sqrt{N}_{a}\right) / 2=5.06$, which gives a much better agreement with the full bladed disk numerical computations.

\section{CONCLUDING REMARKS}

We have derived a new reduced order model, the AMM (Asymptotic Mistuning Model), for the description of the forced response of a mistuned bladed disk. The AMM has been previously applied to study the stabilizing effect of intentional mistuning on the stability characteristics of aerodynamically unstable rotors [18].
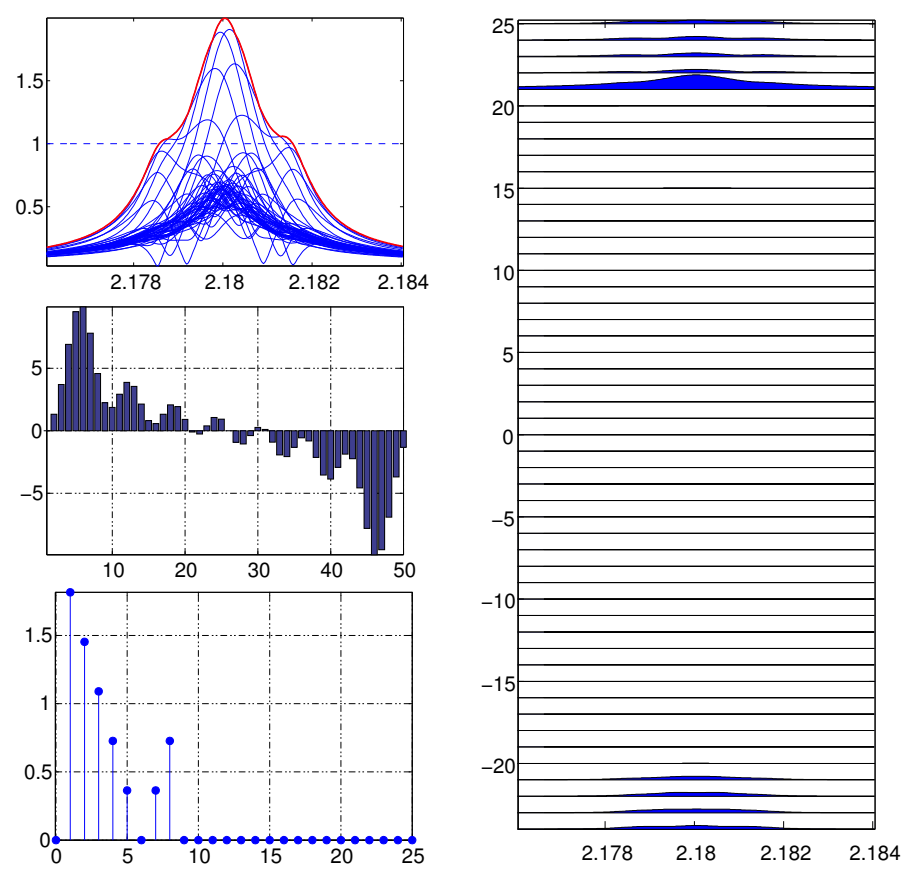

Figure 7. Response of the system in Fig. 2 to a forcing with engine order 21 (tuned TW frequency $\omega_{0}=2.1830 \ldots$ ) and mistuning pattern composed of only harmonics with wavenumber 1 to 8 (AMM prediction for maximum response). Top left: displacements $\left|x_{j}\right|$ vs. forcing frequency $\left(\max \left|x_{j}\right|=1.991 \ldots\right)$. Middle left: mistuning distribution $\delta_{j}$. Bottom left: amplitude of the fourier modes of the mistuning distribution. Right: amplitude of the TW components of the response vs. forcing frequency (wavenumber in the vertical axis).

The AMM captures the essential ingredients that are involved in the mechanism of mistuned response amplification, and can be regarded as an extension of the FMM [15-17] that allows to describe the dynamics associated with more general modal families (the FMM can only account for modal families with nearly identical frequencies, see Ref. [16])).

The AMM has been systematically derived from the general equations for the forced response of a mistuned rotor under the only assumption of small mistuning and damping. The AMM has been applied to describe the effect of mistuning in two frequent situations: forced response of a pair of isolated modes, and forced response of a group of modes with close frequencies. In the first case the AMM can be completely analyzed and closed form expressions have been derived for the maximum amplification factor and for its associated mistuning distribution. In the second case the problem has to be solved numerically, but the size of the AMM is much smaller than that of the original problem ( $N$ variables at most). In both cases we have found excellent quantitative agreement between the AMM results and the numerical simulation of the forced response of the mistuned mass-spring system given by Eqn. (33). 

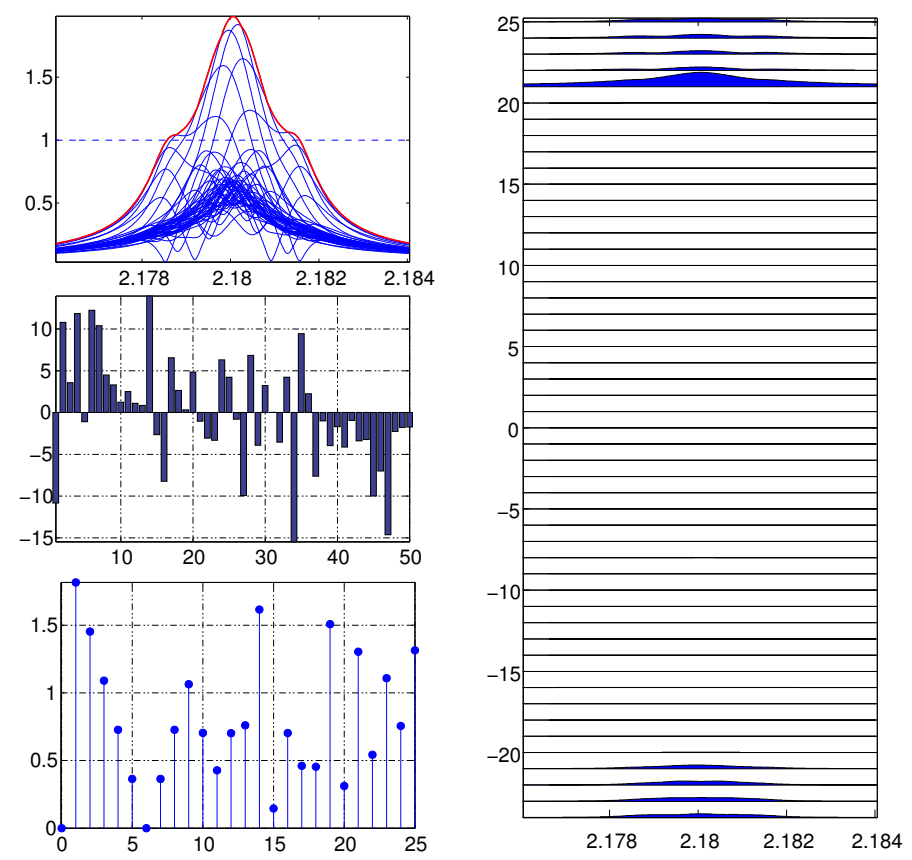

Figure 8. Same as in Fig. 7 but with the rest of the harmonics added to the mistuning pattern with random amplitude $\left(\max \left|x_{j}\right|=1.9851 \ldots\right)$.
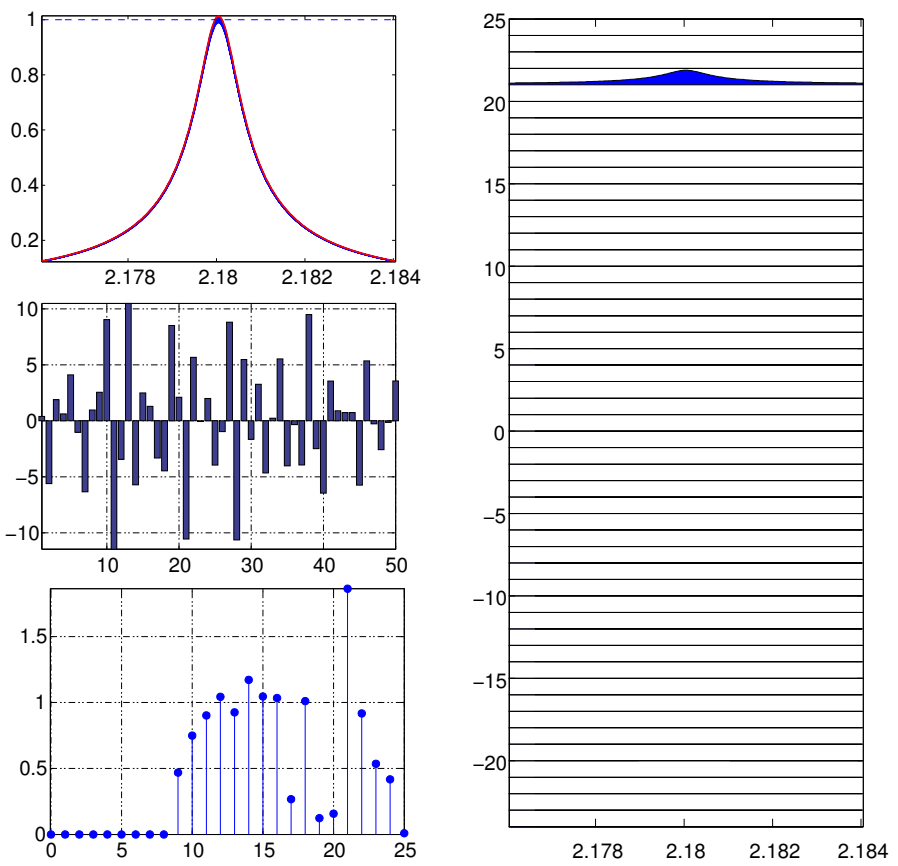

Figure 9. Same as in fig. 7 but for a mistuning pattern with zero harmonics with wavenumber 1 to 8 and the rest with random amplitudes $\left(\max \left|x_{j}\right|=1.0141 \ldots\right)$.
The application of the AMM allows us to draw the following final remarks about the role of mistuning in the near resonance forced response of a bladed disk:

1. The mistuned forced response of the system is composed of only those tuned TW modes that have a frequency that is close the resonant one (the active modes).

2. The effect of the small mistuning is to couple these active TW modes. The coupling between the TWs with wavenumbers $k$ and $k^{\prime}$ takes place through the harmonic of the mistuning distribution with wavenumber $\left(k-k^{\prime}\right)$. The harmonics of the mistuning distribution that have an effect upon the response of the system are only those that couple active modes.

3. The maximum amplification is bounded by

$$
\text { Amplitude }_{\max }=\frac{1+\sqrt{N_{a}}}{2},
$$

where $N_{a}$ is the number of active modes. This constitutes a further refinement of the upper bound obtained by Whitehead [3,22], and it is valid for modal families exhibiting a substantial variation of the natural frequencies. The typical size of the mistuning distribution that gives the maximum amplification is small, of the order of the damping, and depends on the details of the frequency distribution of the active modes.

4. The AMM requires to assume a frequency difference among the active modes of the order of the damping or smaller, and a frequency difference between the rest of the tuned modes and the active modes large as compared with the damping. If separation between the frequencies of the active modes and the rest of the modes is not clear but gradual (in the scale of the damping), then the maximum amplification curve does not exhibit a nice isolated maximum like that in Fig. 3, but the AMM still can be applied to determine the slope and the beginning of the amplification curve if enough modes are retained as active modes.

\section{ACKNOWLEDGMENTS}

The authors wish to thank ITP for the permission to publish this paper and for its support during the project. The work of CM has also been supported by the Spanish Ministerio de Educación y Ciencia under grant MTM2007-62428 and by the Universidad Politécnica de Madrid under grant CCG06-UPM/IME-328. The work of RC has also been supported by the Spanish Ministerio de Educación y Ciencia under grant TRA2006-15015/TAIR. 


\section{REFERENCES}

[1] Slater, J., Minkiewicz, R., and Blair, A., 1999. "Forced response of bladed disk assemblies - a survey". The Shock and Vibration Digest, 31, pp. 17-24.

[2] Castanier, M., and Pierre, C., 2006. "Modeling and analysis of mistuned bladed disk status and emerging directions". Journal of Propulsion and Power, 22(2), pp. 384-396.

[3] Whitehead, D., 1966. "Effect of mistuning on the vibration of turbomachine blades induced by wakes". Journal of Mechanical Engineering Science, 8(1), pp. 15-21.

[4] Han, Y., and Mignolet, M., 2007. "A novel perturbationbased approach for the accurate prediction of the forced response of mistuned bladed disks". presented at the International Gas Turbine Institute Turbo Expo, Montreal, Canada, ASME Paper GT2007-27352.

[5] Han, Y., Xiao, B., and Mignolet, M., 2007. "Expedient estimation of the maximum amplification factor in damped mistuned bladed disks". presented at the International Gas Turbine Institute Turbo Expo, Montreal, Canada, ASME Paper GT2007-27353.

[6] Petrov, E., and Ewins, D., 2003. "Analysis of the worst mistuning patterns in bladed disk assemblies". Journal of Turbomachinery, 125, October, pp. 623-631.

[7] Ewins, D., 1969. "The effects of detuning upon the forced vibrations of bladed-disks". Journal of Sound and Vibration, 9(1), pp. 65-79.

[8] Sinha, A., 1997. "Computation of the maximum amplitude of a mistuned bladed disk assembly via infinity norm". Proceedings of the 1997 ASME International Mechanical Engineering Congress and Exposition, AD-55, pp. 427-432.

[9] Kenyon, J., and Griffin, J., 2003. "Experimental demonstration of maximum mistuned bladed disk forced response". Journal of Turbomachinery, 125, October, pp. 673-681.

[10] Kenyon, J., Griffin, J., and Feiner, D., 2003. "Maximum bladed disk forced response from distortion of a structural mode". Journal of Turbomachinery, 125, April, pp. 352363.

[11] Yang, M., and Griffin, J., 1997. "A reduced order approach for the vibration of mistuned bladed disk assemblies". Journal of Engineering for Gas Turbines and Power, 119, January, pp. 161-167.

[12] Castanier, M., Ottarson, G., and Pierre, C., 1997. "A reduced order modeling technique for mistuned bladeddisks". Journal of Vibration Acoustics, 119(3), pp. 439447.

[13] Yang, M., and Griffin, J., 2001. "A reduced order model of mistuning using a subset of nominal modes". Journal for Gas Turbines and Power, 123, October, pp. 893-900.

[14] Rivas-Guerra, A., and Mignolet, M., 2003. "Maximum amplification of blade response due to mistuning: Localization and mode shape aspects of the worst disks". Journal of Turbomachinery, 125, July, pp. 442-454.
[15] Feiner, D., and Griffin, J., 2002. "A fundamental model of mistuning for a single family of modes". Journal of Turbomachinery, 124, October, pp. 597-605.

[16] Feiner, D., and Griffin, J., 2004. "Misuning identification of bladed disks using fundamental mistuning modelpart i: Theory". Journal of Turbomachinery, 126, January, pp. $150-158$.

[17] Feiner, D., and Griffin, J., 2004. "Misuning identification of bladed disks using fundamental mistuning model-part ii: Application". Journal of Turbomachinery, 126, January, pp. 159-165.

[18] Martel, C., Corral, R., and Llorens, J., 2008. "Stability increase of aerodynamically unstable rotors using intentional mistuning". Journal of Turbomachinery, 130, January, p. to appear.

[19] Wilkinson, J., 1988. The Algebraic Eigenvalue Problem. Oxford Science Publications.

[20] MacBain, J., and Whaley, P., 1984. "Maximum resonant response mistuned bladed disks". Journal of Vibration, Acoustics, Stress and Reliability in Design, 106, April, pp. 218-223.

[21] Kenyon, J., and Griffin, J., 2003. "Forced response of turbine engine bladed disks and sensitivity to harmonic mistuning". Journal of Turbomachinery, 125, January, pp. 113-120.

[22] Whitehead, D., 1998. "The maximum factor by which forced vibration of blades can increase due to mistuning". Journal of Turbomachinery, 120, January, pp. 115-119. 